\title{
ARTICLE
}

\section{Upper gastrointestinal tract tumours: diagnosis and staging strategies}

\author{
Richard M Gore \\ Department of Radiology, Evanston Northwestern Healthcare, Northwestern University, 2650 Ridge Ave, \\ Evanston, IL 60201, USA \\ Corresponding address: Richard M Gore, MD, Gastrointestinal Imaging, Department of Diagnostic Radiology, \\ Evanston Northwestern Healthcare, Northwestern University, 2650 Ridge Avenue, Evanston, IL 60201, USA. \\ E-mail: rmgore1953@aol.com
}

Date accepted for publication 13 April 2005

\begin{abstract}
In patients with oesophageal and gastric cancer, accurate assessment of tumour extent within and beyond the gut wall and detection of lymph node and distant metastases are of paramount importance in planning the surgical approach, in deciding whether neo-adjuvant chemotherapy or radiation therapy is necessary, and in determining the risk of tumour recurrence and overall prognosis. The utility of MDCT, MR, endoscopic ultrasound, PET, PET/CT is discussed and recommendations for cost-effective imaging in these patients are presented.
\end{abstract}

Keywords: Stomach CT; oesophagus CT; endoscopic ultrasound; stomach cancer; oesophagus cancer.

\section{Introduction}

Cancers of the oesophagus and stomach are among the most lethal of all malignancies. The majority of these neoplasms in Western countries are detected at an advanced stage due to the insidious nature of the onset of symptoms and their similarity in early stages to benign causes of dysphagia and dyspepsia. Only by earlier diagnosis, more accurate staging methods, and more effective treatment protocols can we offer any hope of improving the dismal prognosis of these tumours.

\section{Oesophageal cancer}

In the past, squamous cell carcinoma accounted for over $95 \%$ of oesophageal malignancies. Over the past two decades, however, there has been a dramatic increase of adenocarcinoma arising in columnar celllined Barrett's mucosa, accounting for greater than $50 \%$ of all oesophageal cancers in some areas ${ }^{[1]}$.

\section{Diagnosis}

On double-contrast barium studies, early squamous cell carcinomas of the oesophagus appear as small, sessile, polypoid lesions, with smooth or slightly lobulated contours; or as plaque-like lesions that often have flat, central ulcers that are best visualized in profile; or as a superficial, spreading lesion with a nodular appearance of the mucosa without a discrete mass. When early oesophageal cancer or superficial spreading cancer is suspected on barium examinations, endoscopic biopsy should be performed. Advanced squamous cell carcinomas may appear infiltrative, ulcerative, polypoid or less commonly varicoid.

Early adenocarcinoma arising from Barrett's mucosa can manifest as small sessile polyps, plaque-like lesions, or superficial spreading lesions that cause focal nodularity of the mucosa without a discrete mass. These early cancers can also cause focal irregularity, flattening or nodularity of a pre-existing peptic stricture. Accordingly, early endoscopy and biopsy are necessary to exclude adenocarcinoma whenever any of these

This paper is available online at http://www.cancerimaging.org. In the event of a change in the URL address, please use the DOI provided to locate the paper. 
suspicious features develop in the region of a peptic stricture. Advanced adenocarcinoma of the oesophagus can appear infiltrating, polypoid, ulcerative, or, less commonly varicoid (Fig. 1) ${ }^{[2]}$.

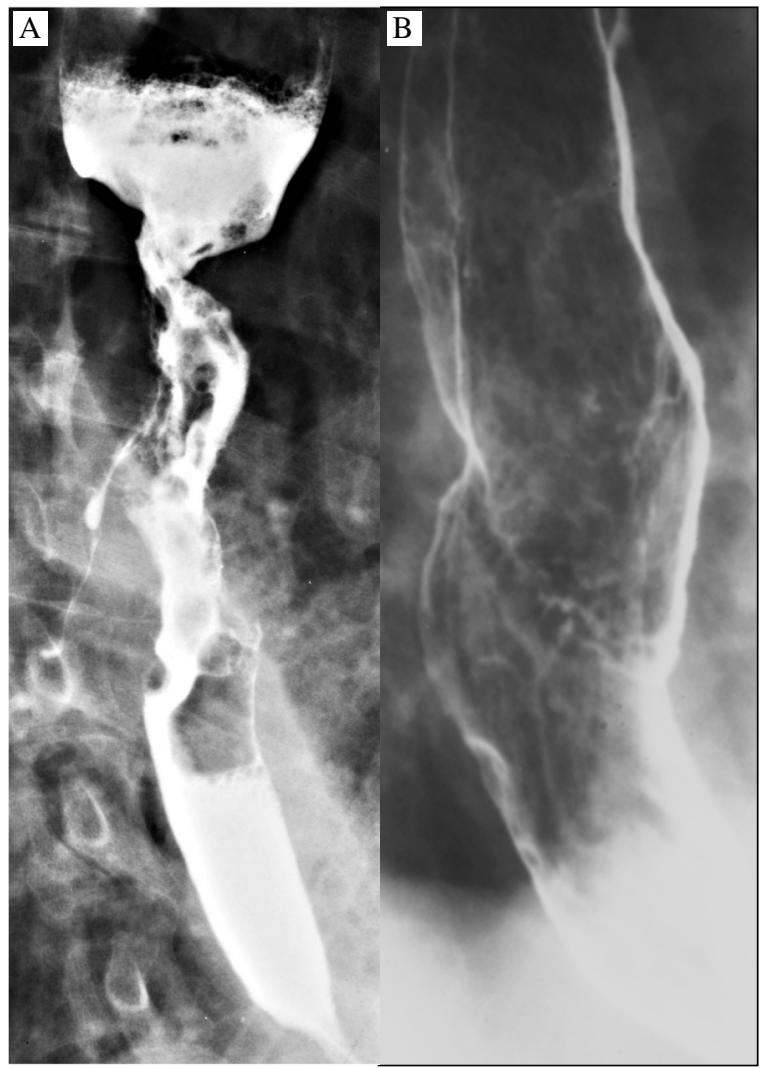

Figure 1 Carcinoma of the oesophagus on double contrast barium studies. (A) Squamous cell carcinoma showing luminal narrowing, abrupt shelf-like borders, ulceration, circumferential growth and a fistula to the tracheobronchial tree. (B) Adenocarcinoma arising from Barrett's mucosa causes a benignappearing stricture associated with a plaque-like tumour of the mid-oesophagus.

\section{Gastric cancer}

Cancers of the antrum and body of the stomach have decreased in incidence in Western countries but the incidence of adenocarcinomas at the gastro-oesophageal junction have been dramatically rising. Early gastric cancer can only be found by screening asymptomatic, atrisk patients.

\section{Diagnosis}

Early gastric cancer is limited to the mucosa and submucosa, regardless of the presence or absence of lymph node involvement. Type 1 early gastric cancers are elevated lesions that protrude more than $5 \mathrm{~mm}$ into the lumen. Type 2 tumours appear as plaque-like elevations with mucosal nodularity, or shallow areas of ulceration, singly or severally. Type 3 early gastric cancers are excavated lesions resembling gastric ulcers but with irregular ulcer craters, clubbing, fusion, or amputation of radiating folds, and nodularity of adjacent mucosa.

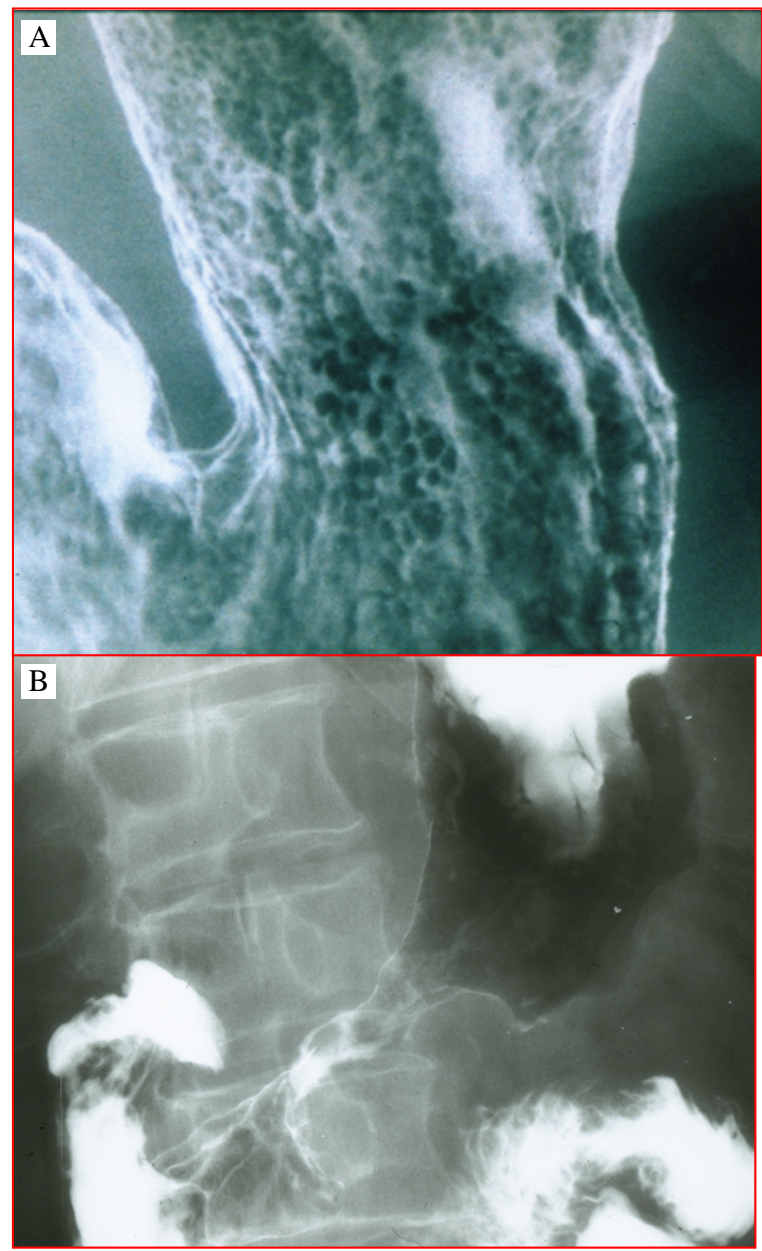

Figure 2 Adenocarcinoma of the stomach. (A) Early gastric cancer with mucosal nodularity. (B) Advanced gastric cancer with narrowing and rigidity of the antral wall due to mural infiltration of scirrhous tumour.

Type 1 advanced gastric cancer is a large polypoid or fungating lesion that has irregular lobulation and measures $3 \mathrm{~cm}$ or larger in greatest diameter. In Type 2 advanced gastric cancer, the bulk of the tumour has been replaced by ulceration. These tumours have discrete, sharply defined borders. Type 3 advanced gastric cancers have mixed morphology with both infiltrative and ulcerative components. The ulceration does not have discrete borders, however. Type 4 advanced gastric cancers are diffusely infiltrating lesions that are associated with marked proliferation of fibrotic tissue and desmoplasia producing the so-called linitis plastica appearance (Fig. 2) ${ }^{[3]}$. 


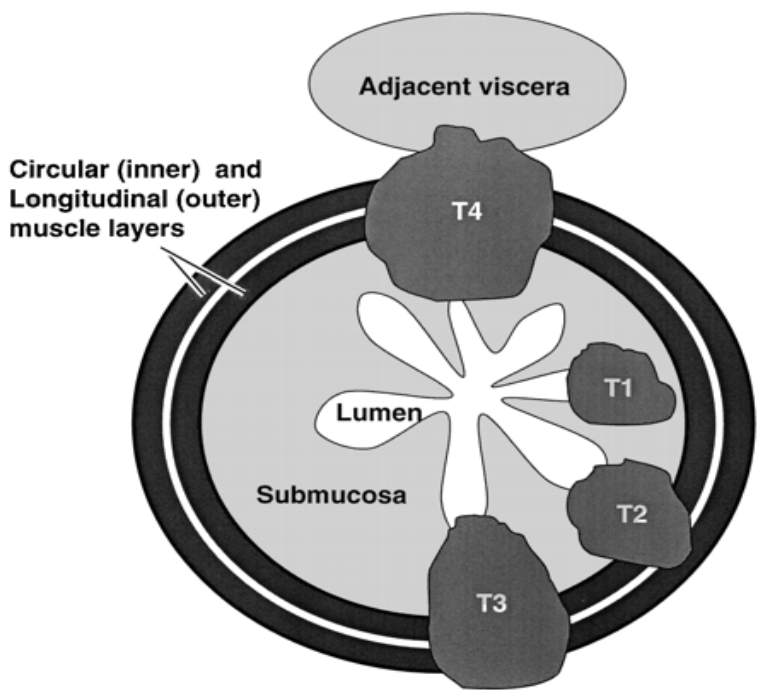

Figure 3 Schematic showing $T$ staging of oesophageal and gastric cancer. T1, tumour extends into submucosa; T2, tumour extends into muscularis propria; T3, tumour extends through the muscularis propria into the subserosa; T4, tumour extends directly into other organs or tissues.

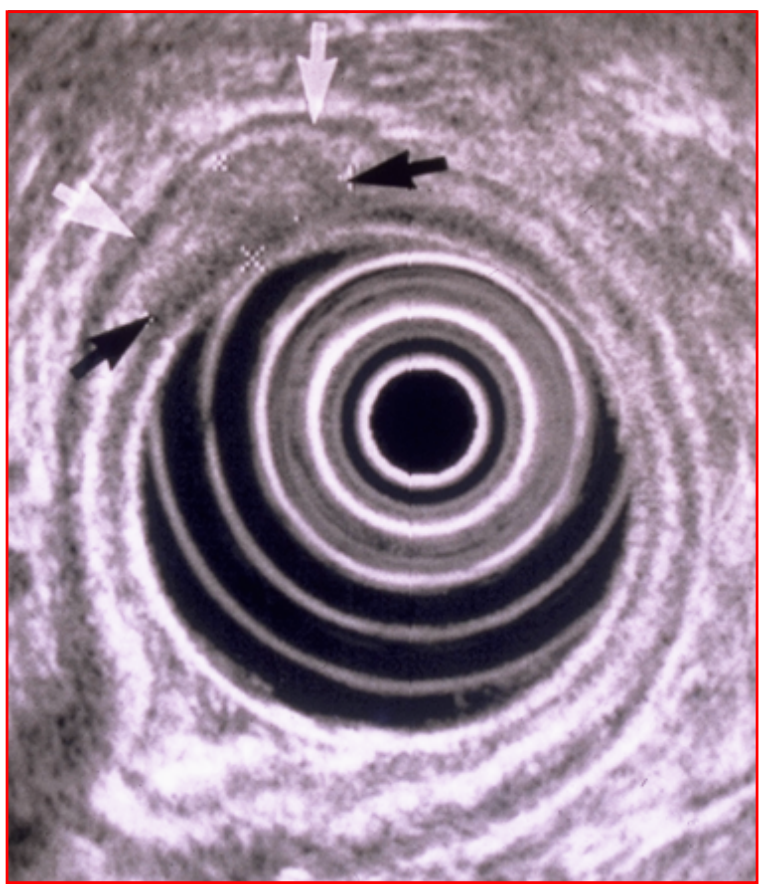

Figure 4 Endoscopic ultrasound demonstrates a T2 oesophageal neoplasm that has invaded but has penetrated beyond the muscularis propria.

\section{Staging}

Once the diagnosis of oesophageal and gastric cancer is established, accurate staging is essential in planning the surgical approach, in deciding whether neoadjuvant chemotherapy or radiation therapy is necessary, and in determining the risk of tumour recurrence and overall prognosis.

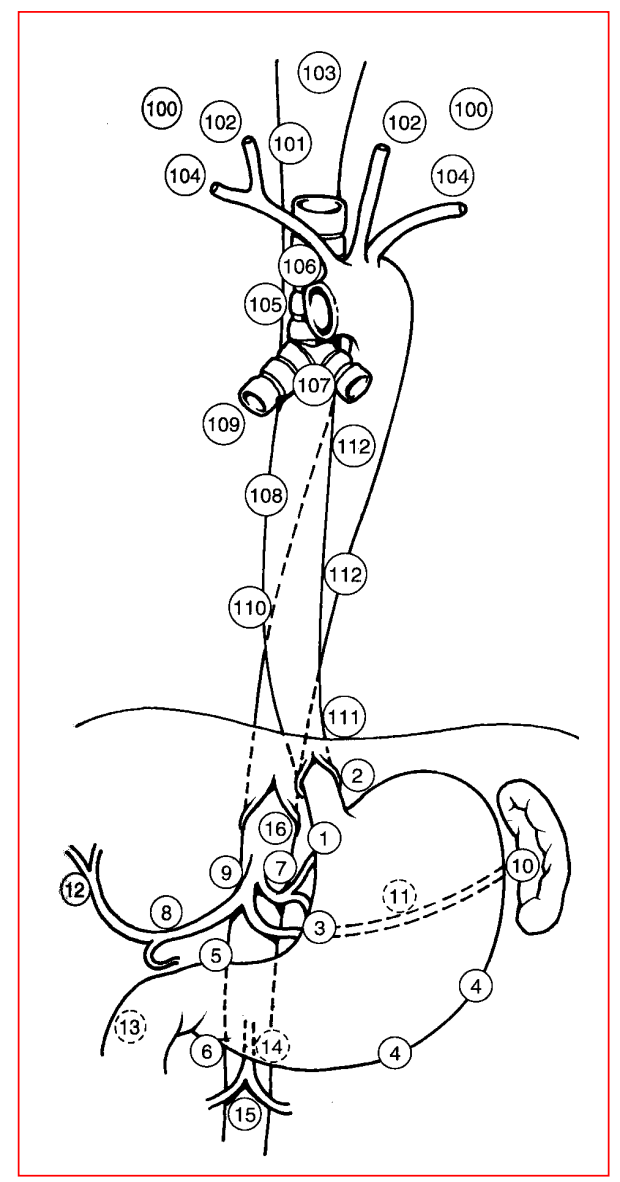

Figure 5 N staging of oesophago-gastric neoplasm. Schematic diagram depicting common sites of lymph node metastases in oesophageal and gastric cancers.

A number of imaging examinations have proven useful for upper gastrointestinal tumour staging ${ }^{[4-8]}$ :
(1) MDCT
(2) MRI
(3) endoluminal MRI
(4) transabdominal ultrasound
(5) endoscopic ultrasound
(6) intraoperative ultrasound
(7) PET
(8) PET/CT.

\section{T staging}

$\mathrm{T}$ staging assesses the depth of tumour invasion into the wall of the oesophagus and stomach, surrounding adventitia, serosa, fat, and adjacent organs (Fig. 3). Endoscopic ultrasound is superior to endoscopic MR in 
depicting the depth of mural invasion for oesophagogastric neoplasms and both modalities are superior to MDCT and conventional MR. PET and PET/CT have only a limited role in this aspect of tumour staging (Fig. 4).

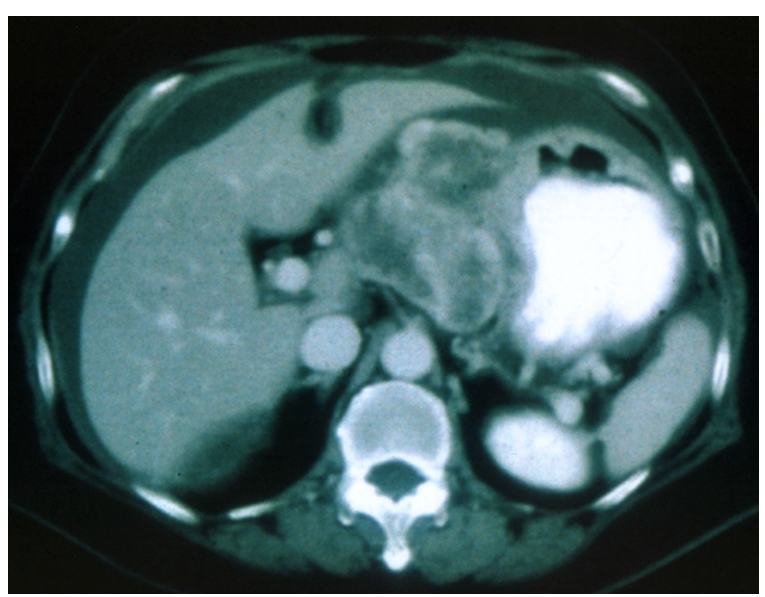

Figure 6 CT scan shows extensive adenopathy in the gastro-hepatic ligament due to spread of an adjacent gastric neoplasm.

\section{$N$ staging}

CT and MR detection of malignant lymphadenopathy has traditionally been based on size criteria. Lymph nodes greater than $1 \mathrm{~cm}$ are considered abnormal. Unfortunately size criteria are based only on statistical probability. In reality, many nodes smaller than $1 \mathrm{~cm}$ are malignant, and nodes larger than $1 \mathrm{~cm}$ are caused by reaction to a number of benign inflammatory conditions. Accordingly, CT and MR cannot reliably differentiate benign from malignant adenopathy (Figs. 5 and 6).

Endoscopic ultrasound is superior to MDCT, conventional MR and endoscopic MR in the depiction of local adenopathy. PET/CT is superb for detecting regional and distant adenopathy.

\section{$M$ staging}

Once oesophageal and gastric cancer have become invasive, there are five major routes of metastases that can be assessed with imaging: (1) direct invasion; (2) lymphatic permeation and dissemination; (3) haematogenous embolization; (4) transperitoneal seeding; (5) intraluminal implantation.
MDCT is the standard means of $\mathrm{M}$ staging in most situations. It is superior to MR in depicting mediastinal, hilar, pulmonary, pericardial, pleural, omental, mesenteric and peritoneal disease. PET/CT appears to be the most accurate means of globally evaluating the chest and abdominal cavities for metastatic tumour. Intraoperative ultrasound appears to be the most sensitive technique in the depiction of liver metastases (Fig. 7).

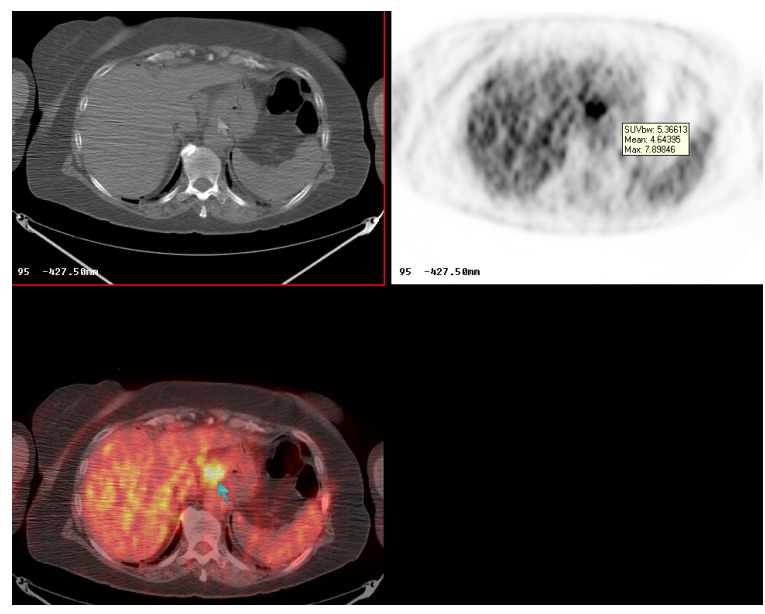

Figure 7 PET scan showing a tumour of the oesophago-gastric junction with metastatic disease to the liver.

\section{References}

[1] Gore RM, Yaghmai, Berlin JW, Newmark G. Oesophageal cancer. In: Imaging in Oncology, 2nd edn. Husband JE, Reznek RH, eds. London: Taylor and Francis, 2004: 159-88.

[2] Gore RM, Yaghmai V. Esophageal cancer. In: Oncologic Imaging, 2nd edn. Bragg DG, Rubin P, Hricak H, eds. Philadelphia: Saunders, 2002: 359-90.

[3] Gore RM, Miller FH. Stomach cancer. In: Oncologic Imaging, 2nd edn. Bragg DG, Rubin P, Hricak H, eds. Philadelphia: Saunders, 2002: 391-418.

[4] Dave UR, Williams AD, Wilson JA et al. Esophageal cancer staging with endoscopic MR imaging. Radiology 2004; 230: 281.

[5] Weber WA, Ott K. Imaging of esophageal and gastric cancer. Semin Oncol 2004; 31: 530-41.

[6] Abdalla EK, Pisters PW. Staging and preoperative evaluation of upper gastrointestinal malignancies. Semin Oncol 2004; 31 : 513-29.

[7] Fusaroli P, Caletti G. Endoscopic ultrasonography: current clinical role. Eur J Gastroenterol Hepatol 2005; 17: 293-301.

[8] Kato H, Miyazaki T, Nakajima M et al. The incremental effect of positron emission tomography on diagnostic accuracy in the initial staging of esophageal carcinoma. Cancer 2005; 103: 148-56. 